\title{
Right-sided endocarditis involving both tricuspid and pulmonary valves in a patient with ventricular septal defect
}

\author{
G. D. G. OAKLEY, P. H. M. CARSON, AND J. M. SANDERSON \\ From North Staffordshire Hospital Centre, Stoke-on-Trent, Staffordshire
}

$A$ patient with alpha-haemolytic streptococcus endocarditis on a ventricular septal defect is described. The disease spread to involve both tricuspid and pulmonary valves which were largely destroyed. The patient developed severe right heart failure with pronounced rise in right atrial pressure. This led to right-to-left shunting through the foramen ovale with systemic embolization. She was successfully treated by operative closure of the defects and double right-sided valve replacement.

The pathology and clinical features of right-sided endocarditis are very different from its left-sided counterpart. In addition to posing problems of diagnosis and treatment, pure right-sided endocarditis may give rise to unfamiliar and interesting haemodynamic situations. The following case illustrates these points.

\section{Case history}

A married woman, now aged 27 years, presented to the Department of Cardiology in September 1974 during her second pregnancy. She was known to have had a murmur from childhood but was asymptomatic. Physical examination showed a healthy woman with a harsh pansystolic murmur maximal at the left sternal edge. A clinical diagnosis of a small ventricular septal defect was made and in February 1975 she underwent full-term normal delivery under antibiotic cover.

Four months later she was admitted as an emergency. For two weeks she had suffered progressive weakness, malaise, diarrhoea, cough, abdominal swelling, ankle oedema, and shortness of breath. There was no history of recent dental treatment. On examination she was pale, febrile, and extremely unwell. Her pulse was regular at $110 / \mathrm{min}$. The jugular venous pressure was raised $5 \mathrm{~cm}$ above the sternal angle with prominent $v$ waves. The ventricular impulse was diffusely increased and there was a systolic thrill. The pansystolic murmur was unchanged. A prominent early diastolic murmur was heard at the left sternal edge. This was decrescendo in nature and did not vary with respiration. She was tachypnoeic and there were bilateral basal crepitations. Finger clubbing was not noted. Ascites was detected in the abdomen and a grossly enlarged, tense, non-pulsatile liver extended below the umbilicus. Examination of the central nervous system was normal apart from a long-standing divergent squint. Six blood cultures were taken and the patient was started on penicillin 20 megaunits daily and streptomycin $500 \mathrm{mg}$ daily, with digitalis and diuretics.

Initial investigations showed her to have a microangiopathic haemolytic anaemia. (Haemoglobin $6.0 \mathrm{~g} / 100 \mathrm{ml}$, platelets $70000 / \mathrm{mm}^{3}$, reticulocytes 7 per cent. Fibrinogen $80 \mathrm{mg} / 100 \mathrm{ml}$ fibrin degradation products $40 \mu \mathrm{g} / \mathrm{ml}$. Erythrocyte sedimentation rate $136 \mathrm{~mm} / \mathrm{hr}$. Blood urea $25 \cdot 1 \mathrm{mmol} / 1$.) Intravenous mannitol was added to her treatment because the deterioration in renal function was thought to be the result of coagulation in small vessels. Serum complement was persistently low with increasedurinary red cell excretion. Five of the blood cultures grew Streptococcus mitior (an alpha-haemolytic oral commensal). The organism was fully sensitive to penicillin and the streptomycin was stopped. Adequate blood levels of penicillin were achieved for 6 weeks. On this regimen the patient improved and her renal function and blood picture returned to normal. Six carious teeth were initially extracted and subsequently a full dental clearance was undertaken. Five days after admission she developed 
pleuritic chest pain, right calf tenderness, and patchy shadowing in the right lower zone on the chest $x$-ray film. She was treated with heparin. For the next two months she suffered from recurrent chest problems involving mainly the right base.

One month after admission she was still very ill. In view of persisting anaemia and gross hepatomegaly a liver biopsy was performed which showed the changes of chronic venous congestion only. She also had a renal biopsy in view of a chronically raised erythrocyte sedimentation rate and very low serum complement levels. The appearances were compatible with a healing nephritis associated with infective endocarditis.

Three months after admission she was noted to have central cyanosis and finger clubbing. She developed sudden blindness in the right eye caused by a retinal embolus. Her general condition was deteriorating and frusemide, $120 \mathrm{mg}$ daily, was not controlling her fatigue and dyspnoea.

Cardiac catheterization showed a very large right atrium. A right atriogram, though inconclusive, strongly suggested a right-to-left shunt at atrial level. The shunt across the ventricular septal defect was less than 2:1. The ventricular septal defect was crossed. Aortic $\mathrm{O}_{2}$ saturation was 64 per cent. No aortic regurgitation was seen on aortography. Pressures ( $\mathrm{mmHg}$ ): right atrium $\mathrm{a}=10, \mathrm{v}=15$; right ventricle $35 / 5$; pulmonary artery $34 / 5$. Left ventricular end-diastolic pressure 8 . The Figure shows the pressure withdrawal trace from the pulmonary artery to the right atrium.

The clinical picture and catheter data were interpreted as showing severe pulmonary and tricuspid regurgitation with no evidence of left-sided valve dysfunction. The ventricular septal defect was considered haemodynamically insignificant. A patent foramen ovale was probable. She was referred for operation.

Operation: 13 November 1975 . One-hour circula- tory occlusion with deep hypothermia. Operative findings were a patent foramen ovale and a ventricular septal defect measuring $5 \mathrm{~mm} \times 5 \mathrm{~mm}$ in the membranous septum. Both tricuspid and pulmonary valves were largely destroyed and a large defect in the septal cusp of the tricuspid valve was noted. There were lumps of yellowish material hanging from some cusps and strands of this extended $4 \mathrm{~cm}$ into the pulmonary artery. (The valves were sterile on culture. Histology showed some fibrosis with fibrinous thrombus on the valve surfaces. The vegetations contained numerous gram positive cocci.)

Procedure: The defects were closed with sutures. Both valves were totally excised and replaced by Lillehei Kaster prostheses. Postoperatively the patient made a good recovery. She is no longer cyanosed and the jugular venous pressure is normal. The liver is no longer palpable. Her diuretics have been stopped.

\section{Discussion}

Right-sided endocarditis is rare in the absence of predisposing factors. The most common of these are chronic heroin addiction and congenital heart disease (Roberts and Buchbinder, 1972). The organism is frequently a staphylococcus and the clinical course is dominated by septic pulmonary infarction and septicaemia. Right heart failure, cardiac murmurs, clubbing, and systemic emboli are seldom prominent though evidence of nephritis is frequent (Bain et al., 1958). The tricuspid valve is usually involved though occasionally the pulmonary valve is affected as well, or in isolation.

It is well known that isolated tricuspid regurgitation can be tolerated very well if there is no pulmonary hypertension. Patients with traumatic tricuspid regurgitation have survived for many years (Marvin, Schrank, and Nolan, 1973) and total

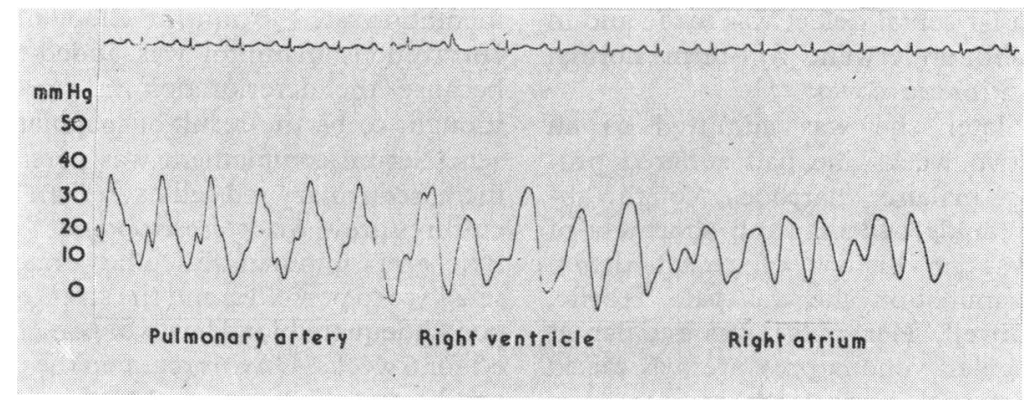

Fig. Pressure withdrawal trace from pulmonary artery through the right ventricle to the right atrium showing the wide pulse pressure in the pulmonary artery and the large systolic waves in the right atrium. 
removal of the tricuspid valve without replacement has been recommended as a life-saving measure in endocarditis associated with heroin addiction (Arbulu, Thoms, and Wilson, 1972). A recent survey, however, showed that 3 patients out of 10 who had this procedure developed right heart failure and it was not possible to identify those at risk from preoperative data (Robin et al., 1975).

Isolated pulmonary regurgitation may also be tolerated well and is compatible with many years of freedom from symptoms (Holmes, Fowler, and Kaplan, 1968; Olesen and Fabricius, 1956). Experiments in dogs support this though the survival of dogs with excised pulmonary valves is very much reduced if there is an associated ventricular septal defect or right ventriculotomy (Ellison et al., 1970). It seems, therefore, that regurgitation or complete absence of either rightsided valve can be well tolerated, but under such circumstances the ventricle is handicapped and little able to cope with further insults. In our case double valve disease constituted such an insult.

The late development of clubbing in our patient was of interest since it coincided with the onset of central cyanosis. The absence of fever, negative blood cultures, and falling erythrocyte sedimentation rate at this time suggested that the clubbing was more likely to be the result of the development of cyanotic heart disease than of persisting infection.

Sporadic reports of right-sided endocarditis on both valves, with a ventricular septal defect, occur in post-mortem series (Boldero and Bedford, 1924). Some haemodynamic data are available on mixed right-sided endocarditis in heroin addicts. Massumi, Just, and Tawakkol (1967) report two such cases, one of whom was living after two years with apparently severe regurgitation of both valves. Surgical intervention has been described in isolated tricuspid endocarditis with a ventricular septal defect (Jimenez-Martinez, Lopez-Cuellar, and QuioñonesMorales, 1971). To our knowledge there has been no report of a ventricular septal defect with rightsided endocarditis on both valves treated by total surgical correction. In studies of necropsy material it was shown that right-sided endocarditis seldom spreads to involve the valve ring and the authors concluded that good results could be expected from operation (Roberts and Buchbinder, 1972). Our case is certainly in accord with this conclusion.

We thank Dr. C. H. J. Swan who originally referred the case and subsequently performed the liver biopsy. We are also indebted to Dr. G. M. Aber and Dr. P. Naish who helped with the management of the renal complications and performed the renal biopsy.

\section{References}

Arbulu, A., Thoms, N. W., and Wilson, R. F. (1972). Valvulectomy without prosthetic replacement: a lifesaving operation for tricuspid Pseudomonas endocarditis. fournal of Thoracic and Cardiovascular Surgery, 64, 103.

Bain, R. C., Edward, J. E., Scheifley, C. H., and Geraci, J. E. (1958). Right-sided bacterial endocarditis and endarteritis. American fournal of Medicine, 24, 98.

Boldero, H. E. A., and Bedford, D. E. (1924). Infective endocarditis in congenital heart disease involving the pulmonary artery. Lancet, $2,747$.

Ellison, R., Brown, W. J., Yeh, T. J., and Hamilton, W. F. (1970). Surgical significance of acute and chronic pulmonary valvular insufficiency. Fournal of Thoracic and Cardiovascular Surgery, 60, 549.

Holmes, J. C., Fowler, N. O., and Kaplan, S. (1968). Pulmonary valvular insufficiency. American fournal of Medicine, 44, 851.

Jimenez-Martinez, M., Lopez-Cuellar, M., and QuioñonesMorales, R. (1971). Isolated tricuspid endocarditis with ventricular septal defect. fournal of Thoracic and Cardiovascular Surgery, 61, 665.

Marvin, R. F., Schrank, J. P., and Nolan, S. P. (1973). Traumatic tricuspid insufficiency. American fournal of Cardiology, 32, 723.

Massumi, R. A., Just, H., and Tawakkol, A. (1967). Pulmonary valvular regurgitation secondary to bacterial endocarditis in heroin addicts. American Heart fournal, 73, 308.

Olesen, K., and Fabricius, J. (1956). Pulmonic valvular regurgitation during twenty-seven years after gonorrheal endocarditis. American Heart fournal, 52, 791.

Roberts, W. C., and Buchbinder, N. A. (1972). Right-sided valvular infective endocarditis. A clinicopathologic study of twelve necropsy patients. American fournal of Medicine, $53,7$.

Robin, E., Thoms, N. W., Arbulu, A., Ganguly S., N., and Magnisalis, K. (1975). Hemodynamic consequences of total removal of the tricuspid valve without prosthetic replacement. American fournal of Cardiology, 35, 481.

Requests for reprints to Dr. Peter Carson, Cardiology Department, City General Hospital, Newcastle Road, Stoke-on-Trent, Staffordshire ST4 6QG. 